\title{
Fujita decomposition on families of abelian varieties
}

\author{
Gian Pietro Pirola ${ }^{1}$
}

Received: 11 January 2021 / Accepted: 13 May 2021 / Published online: 27 May 2021

(c) The Author(s) 2021

\begin{abstract}
Let $F: \mathcal{V} \rightarrow B$ be a smooth non-isotrivial 1-dimensional family of complex polarized abelian varieties and $V_{b}=F^{-1}(b)$ be the general fiber. Let $\mathcal{F}^{1} \subset R^{1} F_{*} \mathbb{C}$ be the associated Hodge bundle filtration, $\mathcal{F}_{b}^{1}=H^{1.0}\left(V_{b}\right)$. Under the assumption that the Fujita decomposition for $\mathcal{F}^{1}$ is non trivial, that is there is a non trivial flat sub-bundle $0 \neq \mathbb{U} \subset \mathcal{F}^{1}$, we show that $V_{b}$ has non-trivial endomorphism: $\operatorname{End}\left(V_{b}\right) \neq \mathbb{Z}$.
\end{abstract}

\section{Introduction}

Given a family of compact Kähler varieties $F: X \rightarrow B$ over an algebraic curve $B$, Fujita decomposition [10,11] gives a splitting of the direct image of relative dualizing sheaf $\pi_{*} \omega_{X \mid B}$ into $\mathcal{U} \oplus \mathcal{A}$, where $\mathcal{U}$ is unitary and flat and $\mathcal{A}$ is ample. The positive part of the theorem had many important applications and improvements, we just mention [15,16,31] and [17, Corollary 3.5]. The unitary part was neglected until the fundamental papers of Catanese and Dettweiler [4-6]. They provided (together with a complete written proof of the decomposition) examples where the unitary part cannot be reduced by a base change to a constant factor. These results have inspired many new researches $[9,13,14,20,24,26]$. This paper is a continuation of [9] where the decomposition was related to the Mumford-Tate group. Here we consider a non isotrivial family of abelian varieties, $F: \mathcal{V} \rightarrow B$, and their 1, 0 Hodge bundle $\mathcal{F}^{1}=\mathcal{H}^{1,0} \subset R^{1} F_{*} \mathbb{C}$. The Fujita decomposition for these bundles can be proved directly or by reduction to suitable family of curves. As an application of two powerful results due to Yves André and respectively to Mikhail Borovoi ([1] and [2]), we prove (see Theorem 2.3) that if the unitary part is non zero, then the abelian variety of the family $V_{b}=F^{-1}(b)$, must have non-trivial endomorphisms. Moreover we show that the Néron-Severi group $N\left(V_{b}\right)$ of $V_{b}$, that is the rational endomorphisms fixed by the Rosati involution, is non trivial (see 2.4 and 2.7).

When we have achieved these results we have been in a mixed mood. The first impression was that "this is the end" of Fujita decomposition for these families, becoming at most a paragraph of a book on the endomorphisms of abelian varieties. Now our thinking is brighter.

This article is dedicated to Fabrizio Catanese

$\triangle$ Gian Pietro Pirola

gianpietro.pirola@unipv.it

1 Dipartimento di Matematica, Università di Pavia, Via Ferrata 5, 27100 Pavia, Italia 
It might really be possible that the study of the unitary factor could be helpful. In Sect. 2 we give a small glimpse of it. We use a result of Ribet [25] on the Mumford-Tate group of abelian varieties and a geometric result of Xiao on fibration of curves [32]. We show (see Theorem (3.6)) that a unitary factor of dimension $g-1$ in a family of Jacobian of curves of genus $g$ is semiample. In other words it becomes trivial after a change of basis.

I would like to thank Bert van Geemen, who many years ago explained to me the basics on the Mumford-Tate group. He also pointed out a mistake in a previous version of this paper. I would also like to thank Alessandro Ghigi for the many useful conversations. It is a great pleasure to dedicate these efforts to Fabrizio, without his work I would not have even thought about these topics.

\section{Fujita decomposition and Hodge classes}

Let $B$ be a smooth connected algebraic complex curve. Let $F: \mathcal{V} \rightarrow B$ be a smooth non-isotrivial 1-dimensional family of complex polarized abelian varieties of dimension $g$, $\operatorname{dim} \mathcal{V}=g+1$, let $V_{b}=F^{-1}(b)$ be the general fiber. Let $\mathbb{K}$ be a commutative ring, here we will consider the cases $\mathbb{K}=\mathbb{Z}, \mathbb{Q}, \mathbb{R}$, and $\mathbb{C}$, let $R^{s} F_{*} \mathbb{K}, s \in \mathbb{N}$, be the local system associated to $H^{s}\left(V_{b}, \mathbb{K}\right)$. The polarization gives a section $\omega$ of $R^{2} F_{*} \mathbb{Z}$. The Hodge bundle $\mathcal{H}^{1}=R^{1} F_{*} \mathbb{C} \otimes \mathcal{O}_{B}$ is equipped with the sub-bundle $\mathcal{F}^{1} \subset \mathcal{H}^{1}$, where $\mathcal{F}_{b}^{1}=H^{1.0}\left(V_{b}\right)$. Scalar extension defines a natural sheaf inclusion $\mathbb{H}^{1}=R^{1} F_{*} \mathbb{C} \subset \mathcal{H}^{1}$. One has the Fujita decomposition:

$$
\mathbb{H}^{1}=(\mathbb{U} \oplus \overline{\mathbb{U}}) \oplus \mathbb{A}
$$

where $(\mathbb{U} \oplus \overline{\mathbb{U}}) \otimes \mathcal{O}_{B}$ is the largest unitary flat bundle of $\mathcal{H}^{1}$, and $\mathcal{U}=\mathbb{U} \otimes \mathcal{O}_{B} \subset \mathcal{F}^{1}$.

Let $k$ be the rank of $\mathcal{U}$. Since the family is not isotrivial then $\mathbb{A} \neq 0$ and therefore $k<g$. One has the filtration $\mathcal{A}^{1.0} \subset \mathcal{A}=\mathbb{A} \otimes \mathcal{O}_{B}$, where $\mathcal{F}^{1} \cap \mathcal{A}=\mathcal{A}^{1.0}$, and a (non holomorphic) decomposition $\mathcal{A}=\mathcal{A}^{1.0} \oplus \mathcal{A}^{0.1}, \mathcal{A}^{0.1}=\overline{\mathcal{A}^{1.0}}$. The polarization gives isomorphisms between the dual $\mathcal{U}^{*}$ of $\mathcal{U}$ and $\overline{\mathbb{U}} \otimes \mathcal{O}_{B}$ (with the anti-linear definition of the tensor) and similarly, between $\mathcal{A}^{1,0^{*}}$, the dual of $\mathcal{A}^{1,0}$, and $\mathcal{A}^{0,1}$.

Remark 2.1 Usually the Fujita decomposition is considered only for top degree holomorphic forms. The use of the same name is motivated by the case of the Jacobians: the Fujita decomposition for a family of smooth curves is isomorphic to the decomposition of $(1,0)$ forms of the corresponding family of Jacobians.

It has been proved in [9] that if $k>0$ the image of the modular map $\mathcal{P}: B \rightarrow \mathcal{A}_{g}$ is contained in a Hodge locus, where $\mathcal{A}_{g}$ is the suitable moduli space of polarized abelian varieties. That is for any $b \in B$ the Mumford-Tate group $G\left(V_{b}\right)$ (see [21] and [12]) is smaller than $\mathbb{C S} P_{g}$, the conformal symplectic group. We recall that $S P_{g}$ is the symplectic rational group with respect to the fixed polarization. Here we give two reasons for this:

1. The basic observation of [9]: The Hodge bundle $\mathcal{H}^{k}=R^{k} F_{*} \mathbb{C} \otimes \mathcal{O}_{B}=\bigwedge^{k} \mathcal{H}^{1}$ contains the line bundle $\mathcal{L}=\wedge^{k} \mathcal{U}$, where $k$ is the rank of $\mathcal{U}$. After a finite base change $\pi: D \rightarrow B$ the pull-back $\mathcal{L}^{\prime}$ of $\mathcal{L}$ becomes trivial [1,7]. If $\mathcal{V}^{\prime}=\pi^{*}(\mathcal{V})$ is the pullback family, one has that $\mathcal{L}^{\prime}$ sits in the image of the map $H^{k}\left(\mathcal{V}^{\prime}, \mathbb{C}\right) \rightarrow R^{k} F_{*}^{\prime} \mathbb{C}$. There is then a proper substructure of the primitive cohomology of $H^{k}\left(V_{b}, \mathbb{C}\right)$ and hence $G\left(V_{b}\right) \neq \mathbb{C} S P_{g}$. We remarked in [9] that this argument extends to higher weight variations of Hodge structure. 
2. Perhaps more naturally, we can consider the projector

$$
p_{\mathbb{U}}: \mathbb{H}^{1} \rightarrow \mathbb{U} \oplus \overline{\mathbb{U}}
$$

Over $b \in B$, it gives the projection $H^{1}\left(V_{b}, \mathbb{C}\right) \rightarrow \mathbb{U}_{b} \oplus \overline{\mathbb{U}}_{b}$, that is represented, via the decomposition $\mathcal{F}^{1}=\mathcal{H}^{1.0} \rightarrow \mathbb{U} \otimes \mathcal{O}_{B}$, by a hermitian form

$$
\Omega_{U, b}=\sqrt{-1} \sum_{i}^{k} \omega_{i, b} \wedge \bar{\omega}_{i, b},
$$

for suitable $\omega_{i, b} \in \mathbb{U}_{b}$, of rank $k, \Omega_{U, b} \in H^{1.1}\left(V_{b}\right)$. Therefore $b \rightarrow \Omega_{U, b}$ defines flat section $\Omega_{\mathbb{U}}$ of $\mathcal{H}^{2}=R^{2} F_{*} \mathbb{C} \otimes \mathcal{O}_{B}$. By construction $\Omega_{\mathbb{U}}$ is monodromy invariant, but in general not defined over $\mathbb{Q}$, as proved by Catanese and Dettweiler [4-6]. Moreover $\Omega_{\mathbb{U}}$ and the polarization $\omega$ are linearly independent. Deligne's theorem [7] shows that the image $S_{b}$ of the restriction $j^{*}: H^{2}(\mathcal{V}, \mathbb{C}) \rightarrow H^{2}\left(V_{b}, \mathbb{C}\right)$ contains $\left[\Omega_{U, b}\right]$ the class of $\Omega_{U, b}$ and the polarization form. Clearly the local system $\mathbb{S}$ of $R^{2} F_{*} \mathbb{C}$ defined by the image of $j^{*}$ is proper subsystem defined over $\mathbb{Q}$ of rank $\geq 2$, hence $G\left(V_{b}\right) \neq \mathbb{C S} P_{g}$.

For any unitary factor $\mathbb{W} \subset \mathbb{U}$ of rank $s \leq k$ the projector $p_{\mathbb{W}}: \mathbb{H}^{1} \rightarrow \mathbb{W} \oplus \overline{\mathbb{W}}$ gives raises to a 1.1 hermitian semidefinite class of rank $s, \Omega_{\mathbb{W}}$ invariant under monodromy. A priori one does not know if $\Omega_{\mathbb{W}}$ is a Hodge class, that is invariant under the Hodge group $H$ (see [21]) of the general fiber. We recall that $G=\mathbb{C} H$, and $H$ is the intersection of the Mumford-Tate group $G$ with the special linear group. Nevertheless the combination of the results ([1] and [2]) due to Yves André and respectively to Mikhail Borovoi gives striking results.

Let $\operatorname{End}\left(V_{b}\right)$ be the endomorphism algebra of $V_{b}$, set $\operatorname{End}^{0}\left(V_{b}\right)=\operatorname{End}\left(V_{b}\right) \otimes \mathbb{Q}$ and let $N\left(V_{b}\right) \subset E n d^{0}\left(V_{b}\right)$ be the algebra fixed by the Rosati involution, $N\left(V_{b}\right)$ is the space of rational 1, 1-cycles, that is the rational Néron-Severi group of $V_{b}$.

Definition 2.2 Let $\Pi_{1}(B, b)$ be the fundamental group of $B$ with base point $b \in B, \rho$ : $\Pi_{1}(B, b) \rightarrow \operatorname{End}\left(H^{1}\left(V_{b}, \mathbb{C}\right)\right)$ be the action the monodromy representation, $\rho$ preserves the symplectic form, that is the polarization of $V_{b}$. Let $M$ be the connected component of the Zariski closure of $\rho\left(\Pi_{1}(B, b)\right)$.

One has that $M \subset H$ and moreover from [1] that $M$ is a normal subgroup of the commutator subgroup $H^{\prime}=(H, H)$ of $H$.

Theorem 2.3 Let $V_{b}$ be the general fiber of $b \in B$. If $\mathbb{U}$ is not trivial, i.e. $k>0$, then $\operatorname{End}\left(V_{b}\right) \neq \mathbb{Z}$, that is $\operatorname{End}^{0}\left(V_{b}\right) \neq \mathbb{Q}$.

Proof Let $H$ be the Hodge group (see [21]) of the general fiber $V_{b}$ of the map $F$ and $H^{\prime}=$ $(H, H)$ be the commutator subgroup, $M$ is a normal subgroup of $H^{\prime}$. Assume by contradiction that $E n d^{0}(A)=\mathbb{Q}$, then by [2] the Hodge group $H$ is $\mathbb{Q}$-simple and then $M=H^{\prime}=H$. Since $\mathbb{U}$ is not zero then $\Omega_{\mathbb{U}} \neq 0$ is invariant by $M=H$. It follows that $\Omega_{\mathbb{U}}$ is a real Hodge cycle. Since the space of the 1,1 Hodge real cycles is $N\left(V_{b}\right) \otimes \mathbb{R}$ and $<\omega, \Omega_{\mathbb{U}}>\subset N\left(V_{b}\right) \otimes \mathbb{R}$ we obtain that $\mathbb{Q} \neq N\left(V_{b}\right) \subset E n d^{0}\left(V_{b}\right)$ and then a contradiction.

We will give a more precise result. 
Theorem 2.4 If $\mathbb{U}$ is not trivial, then the Néron-Severi group of $V_{b}, N\left(V_{b}\right)$ is non trivial, and the form $\Omega_{\mathbb{U}}$ is a real Hodge cycle: $\Omega_{\mathbb{U}} \in N\left(V_{b}\right) \otimes \mathbb{R}$.

The proof follows essentially from the mentioned papers $[1,2]$, here we will give some detail.

Lemma 2.5 The algebraic monodromy group $M$ is a normal subgroup of the Hodge group $H$.

Proof The group $M$ is normal in $H^{\prime}=(H, H)$. If $Z(H)$ is the center of $H$ we have $H^{\prime}$. $Z(H)=H$, then $M$ is normal in $H$. For, fix $g \in H$ and write $g=h z \in H, h \in H^{\prime}$ and $z \in Z(H)$. If $m \in M$ we have $g^{-1} m g=z^{-1} h^{-1} m h z=h^{-1} m h \in M$.

Lemma 2.6 The sub-representation $\mathbb{U} \oplus \overline{\mathbb{U}}$ is the largest $M$-unitary sub-representation of $\mathbb{H}^{1}$. Moreover $\mathbb{U} \oplus \overline{\mathbb{U}}$ is a representation of the Hodge group $H$ (and of $G$ ).

Proof The first part is the definition of the Fujita decomposition. We add some words to see that the largest unitary sub-representation has actually this expression.

Let $H$ be a group and $M \triangleleft H$ be a normal subgroup. Let $V$ be a complex $H$-finite dimensional representation. This induces a $M$-representation. Let $\rho: G \rightarrow G L(V)$ and $\rho_{M}: M \rightarrow G L(V)$ be the induced map, we write $g v=\rho(g) v$. Let $W \subset V$ be a subrepresentation, we say that $W$ is unitary if there is an hermitian form on $W\langle$,$\rangle such that$ $\langle m v, m w\rangle=\langle v, w\rangle \forall v, w \in W$. We remark that $W$ is unitary $\Longleftrightarrow \rho(M)$ is bounded in $G L(V)$, therefore if $W_{1}$ and $W_{2}$ are unitary also $W_{1}+W_{2}$ is unitary. We can define the largest unitary $M-$ sub-representation $W_{\max } \subset V$ of $V$. Let $\rho_{\max }: M \rightarrow G L\left(W_{\max }\right)$ be the associated map. For any $g \in H g W_{\text {max }}$ is again a unitary $M$-sub-representation, in fact since $M$ is normal, for $m \in M$ and $w \in W_{\max }$ we have: $\left.m g w=g\left(g^{-1} m g\right)\right) w=g m^{\prime} w \in g W_{\max }$. This gives that $g W_{\max }$ is a $M$ representation. Moreover if $\rho_{\max }(M) \subset G L(W)$ is bounded and then $\rho_{\max }(g M)$ is also bounded. This gives $g W_{\max }=W_{\max }: W_{\max }$ is a $H$-representation.

Now we come back to our our case: $H$ is the Hodge group, $M$ the monodromy group, and $V=H^{1}$. Let $\mathbb{U}_{\max }$ be the largest unitary representation of $\mathbb{H}^{1}$. We first have $\mathbb{U} \oplus \overline{\mathbb{U}} \subset$ $\mathbb{U}_{\max }$ : the hermitian form on $\mathbb{U}$ is given by the polarization $\omega$. Next we observe that a subrepresentation of $\mathbb{A}$ cannot be unitary (this can be detected using the Higgs field, that is by the infinitesimal variation of the Hodge structure [19] and [29],) it follows $\mathbb{U}_{\max } \cap \mathbb{A}=\{0\}$ and therefore $\mathbb{U}_{\max }=\mathbb{U} \oplus \overline{\mathbb{U}}$.

By the above discussion $\mathbb{U} \oplus \overline{\mathbb{U}}$ is also a $H$-representation.

Proof (of theorem 2.4) Keep the previous notation, we have $\mathbb{L}=\mathbb{U} \oplus \overline{\mathbb{U}}$, and $\mathbb{U}$ are associated to $H$-representations. Then their projectors commute with the $H$ action. It follows (see [21] and [2]) that its class $\left[\Omega_{\mathbb{U}}\right]$ belongs to $N\left(V_{b}\right) \otimes \mathbb{R}$. Then the rank of $N\left(V_{b}\right)$ is bigger than 1 , it follows that $N\left(V_{b}\right) \neq \mathbb{Q}$.

From [2, Lemma 2], when $V_{b}$ is a simple abelian variety we have a stronger result.

Theorem 2.7 Let $F: \mathcal{V} \rightarrow B$ be a smooth non-isotrivial 1-dimensional family of complex abelian polarized varieties of dimension $g$. Assume that $V_{b}=F^{-1}(b)$ is a simple abelian variety. Let $K$ be the center of $\operatorname{End}^{0}\left(V_{b}\right)$ and $K_{0} \subset K$ be the field fixed by the Rosati involution, let $\left[K_{0}: \mathbb{Q}\right]=r$ be its degree. If $\mathbb{U} \neq 0$ then $r>1$. 
Proof Let $G$ be the Mumford-Tate group of $V_{b}$. Any irreducible $G$ sub-representation of $\mathbb{A}$ is not equivalent to any $G$ sub-representation of $\mathbb{U} \oplus \overline{\mathbb{U}}$. Set $H_{\mathbb{R}}^{1}=H^{1}\left(V_{b}, \mathbb{R}\right)$, let $\rho_{\mathbb{R}}: H \rightarrow$ $\operatorname{End}\left(H^{1}\right)$ and $\rho_{M \mathbb{R}}: M \rightarrow \operatorname{End}\left(H_{\mathbb{R}}^{1}\right)$ be the real representations. The mentioned [2, Lemma 2] gives a decomposition

$$
\mathbb{H}^{1}=\bigoplus_{i}^{r} \mathbb{W}_{i}^{k_{i}}
$$

where $r=\left[K_{0}: \mathbb{Q}\right]$, and the $\mathbb{W}_{i} \neq 0$ are real irreducible representation of $M$, pairwise non-equivalent. Then after a rearrangement of the index we may write

$$
\mathbb{U} \oplus \overline{\mathbb{U}}=\bigoplus_{i}^{s} \mathbb{W}_{i}^{k_{i}}, \quad \mathbb{A}=\bigoplus_{j=s+1}^{r} \mathbb{W}_{j}^{k_{j}}
$$

where $0<s<r$ and then $r>1$.

\section{The case $\mathbf{k}=\mathbf{g}-1$}

We keep the previous notation. Let $F: \mathcal{V} \rightarrow B$ be a smooth non-isotrivial 1-dimensional family of complex polarized abelian varieties of dimension $g$. Let $j: B \rightarrow B^{\prime}$ be the completion of $B, B^{\prime}$ is a compact Riemann surface. Under the inclusion of the fundamental group $j_{*}: \pi_{1}(B) \rightarrow \pi_{1}\left(B^{\prime}\right)$ we let $K_{\infty}=$ ker $j_{*}$ be the kernel of $j_{*}$.

Definition 3.1 We will say that the action of the monodromy $M$ is unipotent at $\infty$ if the induced representation $\rho: K_{\infty} \rightarrow \operatorname{End}\left(\mathbb{H}^{1}\right)$ is unipotent.

We remark that if the action of $M$ is unipotent at $\infty$ the unitary bundle $\mathbb{U}$ extends to $B^{\prime}$. We will prove the following:

Theorem 3.2 If $F$ is not isotrivial, $V_{b}$ is simple for a general $b \in B$, and $\operatorname{rank}(\mathbb{U})=\mathrm{g}-1>0$, then $g \geq 8$.

Proof We first give the following:

Lemma 3.3 Under the hypothesis of Theorem 3.2 there is a base change $D \rightarrow B$ such that:

1. the monodromy is unipotent at $\infty$, (see 3.1);

2. $D$ is complete: if $D \stackrel{j}{\hookrightarrow} D^{\prime}$ is the completion $D=D^{\prime}$;

3. any unitary non-trivial factor $\mathbb{W}$ of the Fujita decomposition on D has rank $>1$.

Proof It is well known (see [27]) that there is a base change $h: D \rightarrow B$ such that the action of the monodromy on $\pi^{*} \mathbb{H}^{1}$ is unipotent at $\infty$. Assuming this we show that $\mathcal{V}$ extends to an abelian family over $D^{\prime}$, let $0 \in D^{\prime} \backslash D$ be a point. Since the local monodromy is trivial on $\mathbb{U}$, the compact part of the semistable limit, is an abelian variety $V_{0}$ of dimension $\geq \operatorname{rank}(\mathbb{U})=g-1$. In fact the elements of $(\mathbb{U} \oplus \overline{\mathbb{U}})_{d}, d \in D$, are invariant by the local monodromy action. This gives $(\mathbb{U} \oplus \overline{\mathbb{U}})_{0} \subset H^{1}\left(V_{0}, \mathbb{C}\right)$. If $\operatorname{dim} V_{0}=g-1$ we get that $H^{1}\left(V_{0}, \mathbb{C}\right)=H^{1}\left(V_{0}, \mathbb{Q}\right) \otimes \mathbb{C}=(\mathbb{U} \oplus \overline{\mathbb{U}})_{0}$ is defined over $\mathbb{Q}$. By monodromy invariance, this would be true for any $d \in D$. Since the general fiber of the family, is simple this is impossible. Then $\operatorname{dim} V_{0}=g$ and $V_{0}$ extends the family of abelian varieties to 0 . Similarly 
if $\mathbb{W} \subset \mathbb{U}$ has rank one, we get that up to a base change, $\mathbb{W}$ becomes trivial $[1,7]$. The fixed part theorem would give that $V_{d}$ is not simple.

The statement of the theorem is invariant by a base change and from now on we assume that $F: \mathcal{V} \rightarrow B$ satisfies the three conditions of the above lemma. From Theorem 2.3 we have $K=E n d^{0}\left(V_{b}\right) \neq \mathbb{Q}$ that is $\operatorname{dim}_{\mathbb{Q}} K=e>1$. We now show the following:

Proposition 3.4 Assume $k=\operatorname{dim} \mathbb{U}=g-1$. Let $b \in B$ be the very general point. Then the algebra $K=\operatorname{End}^{0}\left(V_{b}\right)$ is a totally real field of degree $e=[K: \mathbb{Q}]>1$.

Proof Consider the action of the endomorphism on $\mathbb{A}$. The pull-back $\phi \rightarrow \phi^{*}$ defines a map $\psi: K \rightarrow \operatorname{End}\left(A^{1,0}\right) \cong \mathbb{C}$. The map $\psi$ is injective, otherwise there would be endomorphisms with non trivial kernel and $V_{b}$ would not be simple. It follows that $K$ embeds in $\mathbb{C}$ and therefore $K$ is a field.

To see that the image of $\psi$ is contained in $\mathbb{R}$ write $K=\mathbb{Q}(\phi)$. The $\phi$ gives a flat section of $\operatorname{Hom}\left(R^{1} F_{*} \mathbb{C}, R^{1} F_{*} \mathbb{C}\right)$. Then for a local section $s$ of $\mathcal{A}^{1,0}$ we have $\phi(s)=\lambda s, \lambda \in \mathbb{C}$. The $\lambda$ - eigenspace of $\phi$ defines a flat bundle $\mathbb{V}_{\lambda} \subset R^{1} F_{*} \mathbb{C} \otimes \mathcal{O}_{B}$ such that $\mathbb{V}_{\lambda} \otimes \mathcal{O}_{B} \supset \mathcal{A}^{1,0}$. This implies that $\mathbb{V}_{\lambda} \otimes \mathcal{O}_{B} \supset \mathcal{A} \supset \mathcal{A}^{0,1}$, otherwise since $\operatorname{dim} \mathcal{A}^{1,0}=1$ we would have $\mathcal{A}^{1,0}=\mathbb{V}_{\bar{\lambda}} \otimes \mathcal{O}_{B} \cap \mathcal{A}$ and $\mathcal{A}^{1,0}$ would be a flat bundle. By conjugation we have $\mathcal{A}^{0,1} \subset$ $\mathbb{V}_{\bar{\lambda}} \otimes \mathcal{O}_{B}$. It follows that $\lambda=\bar{\lambda}$, and then $\psi(\phi) \in \mathbb{R}$. From the classification of the algebras of endomorphisms of abelian varieties (see [22, Chapter IV]) we get that $K$ is a totally real field and from Theorem $(2.3)$ that $[K: \mathbb{Q}]=e>1$.

Write again $K=\mathbb{Q}(\phi)$, let $p_{\phi}(t)$ be the characteristic polynomial of $\phi$ and $p_{\min }(t)$ be the minimal polynomial of $\phi$ on $H^{1}\left(V_{b}, \mathbb{C}\right)$ (or $H^{1}\left(V_{b}, \mathbb{Q}\right)$ ). Now $p_{\min }(t)$ is irreducible, rational, it has degree $e$, and $p_{\phi}(t)=p_{\text {min }}^{k}(t)$ where $k e=2 g$. The Hodge decomposition gives $\phi=\phi^{1,0}+\phi^{0,1}, \phi^{1,0} \in \operatorname{End}\left(\mathcal{H}^{1,0}\right)$ and $\phi^{0,1} \in \operatorname{End}\left(\mathcal{H}^{0,1}\right), \phi^{0,1}=\overline{\phi^{1,0}}$. Therefore $p_{\phi}(t)=p_{\phi^{1,0}}(t) p_{\phi^{0,1}}(t)=p_{\phi^{1,0}}(t) \bar{p}_{\phi^{1,0}}(t)$. Then, since all the eigenvalues are real, any eigenspace of $\phi$ has even dimension $k=2 s$ : therefore $g=e s$.

The following proposition complete the proof of the Theorem 3.4.

Proposition 3.5 Write $g=e s, s$ must be even and $s \geq 4$. In particular $g$ is even and $g \geq 8$.

Proof The $\phi$ eigenvector decomposition defines flat sub-local system and $\mathbb{H}^{1}$ :

$$
\mathbb{H}^{1}=\oplus_{i}^{e} \mathbb{V}_{\lambda_{i}}, \quad H^{1.0}\left(V_{b}\right)=\oplus_{i}^{e} V_{\lambda_{i b}}
$$

Set

$$
\mathbb{U}_{i}=\mathbb{V}_{\lambda_{i}} \cap \mathbb{U} \subset \mathbb{U}
$$

and assume that $\mathbb{V}_{\lambda_{1}} \otimes \mathcal{O}_{B} \supset \mathcal{A}^{1.0}$, and then $\mathbb{A} \subset \mathbb{V}_{\lambda_{1}}$. Note that the rank of $\mathbb{U}_{1}$ is $s-1$. Set $\mathbb{L}=\mathbb{U}_{1} \oplus \overline{\mathbb{U}}_{1}$ we have

$$
\mathbb{V}_{\lambda_{1}}=\mathbb{A} \oplus \mathbb{L}
$$

We see that $s>2$. In fact if $s=1 \mathbb{V}_{\lambda_{i}} \subset \mathbb{U} i>1$, and if $s=2$ then $\mathbb{U}_{1}=\mathbb{V}_{\lambda_{1}} \cap \mathbb{U} \subset \mathbb{U}$ in both cases $\mathbb{U}$ would be a rank one local subsystem, this is against our assumption 3 of 3.3 . Now we show that $s$ is even. 
Assume by contradiction that $s=g / e$ is odd. In this case the theorem of Ribet [25, Theorem 1] gives that the Mumford-Tate group, $G=G\left(V_{b}\right)$, is given by the elements that commute with $\phi$ :

$$
G=\{g \in \mathbb{C S p}(2 g): g \circ \phi=\phi \circ g\} .
$$

Now one has that $G \cdot \mathbb{V}_{\lambda_{i}}=H \cdot \mathbb{V}_{\lambda_{i}}=\mathbb{V}_{\lambda_{i}}$ and then

$$
\mathbb{H}^{1}=\mathbb{A} \oplus \mathbb{L} \oplus \bigoplus_{i>1}^{e} \mathbb{V}_{\lambda_{i}},
$$

we find $e+1$ not equivalent $H$ representations, using [25, Sect. 3] or again [2, Lemma 2], we would have $[K: \mathbb{Q}]>e$ and then a contradiction.

The proof of the Theorem 3.4 is now complete.

The following result was our modest motivation:

Theorem 3.6 Assume that $f: \mathcal{C} \rightarrow B$ is a smooth non isotrivial family of curves of genus $g>1$ and $F: J(\mathcal{C}) \rightarrow B$ be the associated Jacobians family. Let $\mathbb{U}$ be the unitary factor in Fujita decomposition of $\mathcal{F}^{1} \subset R^{1} F_{*} \mathbb{C}$. If $k=g-1$ then $g \leq 7$ and $\mathbb{U}$ has finite monodromy. That is up to a finite base change $h: D \rightarrow B$, and completion $j: D \rightarrow D^{\prime}$, $j_{*} h^{*}\left(\mathbb{U} \otimes \mathcal{O}_{D}\right)=\mathcal{O}_{D^{\prime}}^{g-1}$.

Proof From the slope inequality of Xiao [32, Corollary 4] we get $g<8$. If $g<8 J(\mathcal{C})$ cannot be simple by Proposition 3.5. We may assume to have an isogeny $J\left(C_{b}\right) \cong \prod_{i=1}^{r} V_{i}$, where the $V_{i}$ are simple. After a base change $h: D \rightarrow B$, we can make the decomposition invariant by monodromy (this can be proved by observing that the projectors $J\left(C_{b}\right) \rightarrow V_{i}$ can be represented by integers matrices with bounded norm, that is a finite set). Then we find $r$ families of abelian varieties $F_{i}: \mathcal{V}_{i} \rightarrow D$ and $J(\mathcal{C}) \cong \prod_{i=1}^{r} \mathcal{V}_{i}$.

Since $k=g-1$ only one of them, say $\mathcal{V}_{1}$, is not trivial. Then by (3.5) $\operatorname{dim} \mathcal{V}_{1}=1$ and

$$
\prod_{i=2}^{r} \mathcal{V}_{i}=\prod_{i=2}^{r} V_{i} \times D
$$

is a trivial family of dimension $g-1$. The unitary factor is then trivial on $D$ and the original $\mathbb{U}$ on $B$ has finite monodromy.

Remark 3.7 A refinement [18, Proposition 2.8] of the mentioned result of Xiao [32], shows that in the previous theorem one can put $g<7$. We think that is very interesting to consider non isotrivial genus $g$ fibered surface $\pi: \mathcal{C} \rightarrow B$, where $B$ is a projective curve, such that

$$
\pi_{*} \omega_{\mathcal{C} \mid B}=\mathcal{O}_{B}^{g-1} \oplus \mathcal{A} .
$$

It is the case of the fibrations with relative irregularity $g-1$. Examples are only known for $g \leq 4$ (see [33] and [23]). It has been proved (see [3,8]) that if such a fibration of genus 5 or 6 exists, the fibers of $\pi$ would be trigonal curves and when $g=6$ of special Maroni invariant. If $g=6$ using methods from [25,28,30], one could even try to prove that the relative Albanese variety is not simple. In fact the image of the (relative) Albanese map would give a surface $S$ on abelian variety $A$ of dimension 5. The homological class of $S$ should not be obtained by intersections of divisors. This $A$ would be an exotic Albanese variety (see [28]) but this is impossible for a simple abelian variety of dimension 5. Despite these efforts the existence problem is completely open. 
Acknowledgements The author is member of GNSAGA (INDAM) and were supported by MIUR: Dipartimenti di Eccellenza Program (2018-2022)-Dept. of Math. Univ. of Pavia and by PRIN Project Moduli spaces and Lie theory (2017).

Funding Open access funding provided by Università degli Studi di Pavia within the CRUI-CARE Agreement.

Open Access This article is licensed under a Creative Commons Attribution 4.0 International License, which permits use, sharing, adaptation, distribution and reproduction in any medium or format, as long as you give appropriate credit to the original author(s) and the source, provide a link to the Creative Commons licence, and indicate if changes were made. The images or other third party material in this article are included in the article's Creative Commons licence, unless indicated otherwise in a credit line to the material. If material is not included in the article's Creative Commons licence and your intended use is not permitted by statutory regulation or exceeds the permitted use, you will need to obtain permission directly from the copyright holder. To view a copy of this licence, visit http://creativecommons.org/licenses/by/4.0/.

\section{References}

1. Andre, Y.: Mumford-Tate groups of mixed Hodge structures and the theorem of the fixed part. Compos. Math. 82, 1-24 (1992)

2. Borovoi, M.: The Hodge group and the algebra of endomorphisms of an abelian variety in Problems in group theory and homological algebra. Yaroslav. Gos. Univ., Yaroslavl, 124-126 (1981). arXiv: 1310.5236

3. Beorchia, V., Pirola, G.P., Zucconi, F.: Trigonal deformations of rank one and Jacobians. IMRN (2019). https://doi.org/10.1093/imrn/rnz216

4. Catanese, F., Dettweiler, M.: The direct image of the relative dualizing sheaf needs not be semiample. Comptes Rendus Mathématique. Académie des Sciences. Paris 3, 241-244 (2014)

5. Catanese, F., Dettweiler, M.: Vector bundles on curves coming from variation of Hodge structures. Int. J. Math 27, 1640001 (2016)

6. Catanese, F., Dettweiler, M.: Answer to a question by Fujita on Variation of Hodge Structures. Higher Dimensional Algebraic Geometry: In honour of Professor Yujiro Kawamata's sixtieth birthdayAdv. Stud. Pure Math. 74, 73-102 (2017)

7. Deligne, P.: Théorie de Hodge II. Inst. Hautes Études Sci. Publ. Math., 40, 5-57 (1971)

8. Favale, F., Naranjo, J.C., Pirola, G.P.: On the Xiao conjecture for plane curves. Geom. Dedicata 195, 193-201 (2018)

9. Frediani, P., Ghigi, A., Pirola, G. P.: Fujita decomposition and Hodge loci. J. Inst. de Math. Jussieu 19(4), 1389-1408 (2020)

10. Fujita, T.: On Kähler fiber spaces over curves. J. Math. Soc. Japan. 30(4), 779-794 (1978)

11. Fujita, T.: The sheaf of relative canonical forms of a Kähler fiber space over a curve. Proc. Japan Acad. Ser. A Math. Sci. 54(7), 183-184 (1978)

12. van Geemen, B.: Kuga-Satake varieties and the Hodge conjecture. Arith. Geom. Algebraic Cycles 276, 51-82 (1998)

13. Gonzàles-Alonso, V., Stoppino, L., Torelli, S.: On the rank of the flat unitary summand of the Hodge bundle. Trans. Am. Math Soc. 372(12), 8663-8677 (2019)

14. Gonzàles-Alonso, V., Torelli, S.: Families of curves with Higgs field of arbitrary large kernel. to appear on Bull. Lond. Math. Soc

15. Kawamata, Y.: Kodaira dimension of algebraic fiber spaces over curves. Invent. Math. 66(1), 55-71 (1982)

16. Kawamata, Y.: Hodge theory and Kodaira dimension. Adv. Stud. Pure Math. 1. Algebraic Var. Anal. Var. 317-327 (1983)

17. Kollár, J.: Higher direct images of dualizing sheaves. Ann. Math. 123(1), 11-42 (1986)

18. Konno, K.: On the irregularity of special non-canonical surfaces publ. Ryms. Kyoto Univ. 30(1), 671-688 (1994)

19. Hitchin, N.: The self-duality equations on a Riemann surface. Proc. Lond. Math. Soc. (3) 55(1), 59-126 (1987)

20. Xin, L.: Family of curves with large unitary summand in the Hodge bundle. Math. Z. 291, 1381-1387 (2019)

21. Mumford, D.: Families of Abelian varieties in algebraic groups and discontinuos subgroups. Am. Math. Soc. Providence R.I., 347-351 (1966)

22. Mumford, D.: Abelian Varieties. Oxford University Press, Oxford (1970)

23. Pirola, G.P.: On a conjecture of Xiao. J. Reine Angew. Mat. 431, 75-89 (1992) 
24. Pirola, G.P., Torelli, S.: Massey Products and Fujita decompositions on fibrations of curves. Collect. Math. 71, 39-61 (2020)

25. Kenneth, A.: Ribet, Hodge classes of certain types of abelian varieties. Am. J. Math. 105, 523-538 (1983)

26. Rizzi, L., Zucconi, F.: Fujita decomposition and Massey product for fibered varieties. preprint. arXiv:2007.01473

27. Schmid, W.: Variation of Hodge structure: The singularities of the period mapping. Invent. Math. 22, 211-319 (1973)

28. Schoen, C.: Albanese Standard and Albanese exotic varieties. Lond. Math. Soc. (2) 74, 304-320 (2006)

29. Simpson, C.: Higgs bundles and local systems. Publ. Math. IHES 75, 5-95 (1992)

30. Tankeev, S.: Algebraic cycles of simple 5-dimensional abelian varieties. Izv. Akad. Nauk SSSR 45(4), 793-823 (1981)

31. Viehweg, E.: Weak positivity and the additivity of the Kodaira dimension for certain fibre spaces. Adv. Stud. Pure Math. 1. Algebraic Var. Anal. Var. 329-353 (1983)

32. Xiao, G.: Fibered algebraic surfaces with low slope. Math. Ann. 276(3), 449-466 (1987)

33. Xiao, G.: Irregularity of surfaces with a linear pencil. Duke Math. J. 55(3), 596-602 (1987)

Publisher's Note Springer Nature remains neutral with regard to jurisdictional claims in published maps and institutional affiliations. 